\title{
Skin Needling in Combination with Antimicrobial Peptides for the Treatment of Keloids: A Novel Perspective Proposal and Approach for Skin Scars
}

\author{
Claudio Marasca ${ }^{a}$ Angelo Ruggiero $^{a}$ Dario Marasca ${ }^{b}$ Matteo Megna ${ }^{a}$ \\ Gabriella Fabbrocini ${ }^{a}$ \\ a Section of Dermatology, Department of Clinical Medicine and Surgery, University of Naples Federico II,

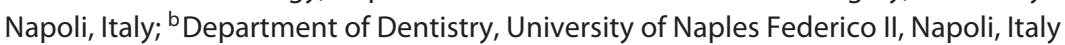

Dear Editor,

We read with great interest the article by Moravvej et al. [1] which reported antimicrobial peptides (AMPs) as a promising alternative for keloid treatment. Specifically, they repurposed AMPs as a promising treatment for fibrotic diseases such as keloids and hypertrophic scars, due to their antifibrogenic properties.

Keloids are fibroproliferative disorders of the skin that are caused by abnormal healing of injured or irritated skin and can be associated with substantial physical and psychological distress [2]. The complex mechanisms leading to the formation of keloids have not yet been fully clarified. While the pathogenesis of keloids continues to be investigated, numerous treatment options exist, but there is still no ideal treatment. Management of keloids needs an association of treatment and long-term followup to observe and manage recurrence [3]. Over the years, many prevention and treatment strategies for keloids have been explored, such as intralesional steroids, silicone-gel sheeting, onion extract, imiquimod $5 \%$ cream, botulinum toxin A, pressure garments, cryotherapy, laser therapy, 5-FU, radiotherapy, bleomycin injection, and surgical excision [4]. In this context, the evidence produced by Moravvej et al. [1] about treatment with AMPs is particularly relevant.
We previously evaluated the individual effectiveness of needling therapy using the Dermapen (Salt Lake City, UT, USA) and topical therapy with silicone gel (Kelocote, Sinclair Pharma, London, UK), and their combined effectiveness for the treatment of linear surgical scars, hypertrophic scars, and keloids [5]. We found that the average improvement was greater when these 2 treatments were combined ( $68 \%$ improvement $[p<0.01]$ on the area treated with a combination of skin needling plus silicone gel vs. $52 \%$ on the area treated with skin needling only, and $63 \%[p<0.01]$ on the area treated with the combination vs. $47 \%$ on the area treated with silicone gel only). Skin needling has proven its efficacy in the treatment of atrophic scars, more than in hypertrophic scars and keloids [6].

In the future, keloids should be treated with combined therapies. We recommend using the new treatment with AMPs proposed by our colleagues, Moravvej et al. [1], in combination with skin needling. We believe that experimental studies are needed in order to evaluate the real efficacy and safety of a treatment based on this promising combination. 


\section{Statement of Ethics}

The study complied with the Declaration of Helsinki. Internal board review number not required.

\section{Conflict of Interest Statement}

The authors have no conflicts of interest to declare.

\section{References}

1 Moravvej $\mathrm{H}$, Memariani $\mathrm{M}$, Memariani $\mathrm{H}$, Robati RM, Gheisari M. Can Antimicrobial Peptides Be Repurposed as a Novel Therapy for Keloids? Dermatology. 2020; DOI: 10.1159/000506831.

2 Ogawa R, Akaishi S, Kuribayashi S, Miyashita T. Keloids and Hypertrophic Scars Can Now Be Cured Completely: Recent Progress in Our Understanding of the Pathogenesis of Keloids and Hypertrophic Scars and the Most Promising Current Therapeutic Strategy. J Nippon Med Sch. 2016;83(2):46-53.
3 Lee HJ, Jang YJ. Recent Understandings of Biology, Prophylaxis and Treatment Strategies for Hypertrophic Scars and Keloids. Int J Mol Sci. 2018;19(3):711.

4 Jaloux C, Bertrand B, Degardin N, Casanova D, Kerfant N, Philandrianos C. Keloid scars (part II): treatment and prevention. Ann Chir Plast Esthet. 2017 Feb;62(1):87-96. French.
5 Fabbrocini G, Marasca C, Ammad S, Brazzini B, Izzo R, Donnarumma M, et al. Assessment of the Combined Efficacy of Needling and the Use of Silicone Gel in the Treatment of C-Section and Other Surgical Hypertrophic Scars and Keloids. Adv Skin Wound Care. 2016 Sep;29(9):408-11.

6 Zaleski-Larsen LA, Fabi SG, McGraw T, Taylor M. Acne Scar Treatment: A Multimodality Approach Tailored to Scar Type. Dermatol Surg. 2016 May;42 Suppl 2:S139-49. 